\title{
MONOLITHIC INTEGRATION OF BINARY-PHASE FRESNEL ZONE PLATE OBJECTIVES ON 2-AXIS SCANNING MICROMIRRORS FOR COMPACT MICROENDOSCOPES
}

\author{
Karthik Kumar ${ }^{1}$, Hung $\mathrm{Cao}^{2}$, and Xiaojing Zhang ${ }^{2}$ \\ ${ }^{1}$ Department of Electrical and Computer Engineering, University of Texas, Austin, Texas, USA \\ ${ }^{2}$ Department of Biomedical Engineering, University of Texas, Austin, Texas, USA
}

\begin{abstract}
We demonstrated unique monolithic integration of Fresnel zone plate objective on 2-axis staggered vertical combdrive micromirror surface via direct patterning of reflective binaryphase-modulation elements on silicon. Need for focusing optics is thus obviated, simplifying microendoscope assembly and improving form factor. Micromirrors scanning $\pm 9^{\circ}$ (optical) about both axes are patterned with elliptical zones designed for $7 \mathrm{~mm}$ (8mm) focal length and $20^{\circ}\left(45^{\circ}\right)$ off-axis 635-nm illumination using 635nm (1310nm) laser. Videos of samples acquired with $\sim 15 \mu \mathrm{m}$ lateral resolution over $1 \mathrm{~mm} \times 0.35 \mathrm{~mm}$ field of view at 5.0 frames/second using the device in a bench-top single-fiber laserscanning confocal microscope confirm applicability of the device to microendoscopy.
\end{abstract}

\section{INTRODUCTION}

In vivo optical imaging is an important tool for medical diagnosis in situations where biopsy is difficult, and for imageguided microsurgery and photodynamic therapy [1]. Cellular-level imaging enables early detection of many diseases such as cancers, which can be crucial to effective treatment and patient survival chances [2]. Visualization in sensitive internal non-tubular human organs requires compact forward-imaging endoscopes, which have thus far proven to be difficult to assemble in small form factor, as opposed to sideways-imaging probes [3]. Microelectromechanical system (MEMS) technologies are uniquely positioned to provide distal beam deflection for image formation in microendoscopes. Vertical combdrive micromirrors have been shown to provide the large rotational torque, deflection angles and mirror surface quality for laser-scanned imaging systems [4]. However, catheter rigid length and outer diameter are mainly limited by focusing optics. Micromachined 3-D optics has been assembled into integrated free-space systems [5]. For multi-element systems, such as catheters, additional assembly becomes increasingly challenging and expensive. The present research addresses the above limitations by monolithically integrating a micromachined Fresnel zone plate objective directly on the surface of the micromirror via patterning of reflective binary-phase-modulation elements.

\section{THEORY}

Coherent illumination incident on the micromirror surface at an angle to the mirror normal can be concentrated at a wavelengthdependent focal distance by an Elliptical Zone Plate (EZP, Figure 1) designed according to equation (1):

$$
\begin{gathered}
\left(\frac{x}{a_{n} \cos \theta}\right)^{2}+\left(\frac{y-b_{n}}{a_{n}}\right)^{2}=1, \text { where } \\
a_{n}=\frac{\sqrt{n \lambda\left(f \cos ^{2} \theta+\frac{n \lambda}{4}\right)}}{\cos ^{2} \theta} \text { and } b_{n}=\frac{n \lambda \sin \theta}{2 \cos ^{2} \theta}
\end{gathered}
$$

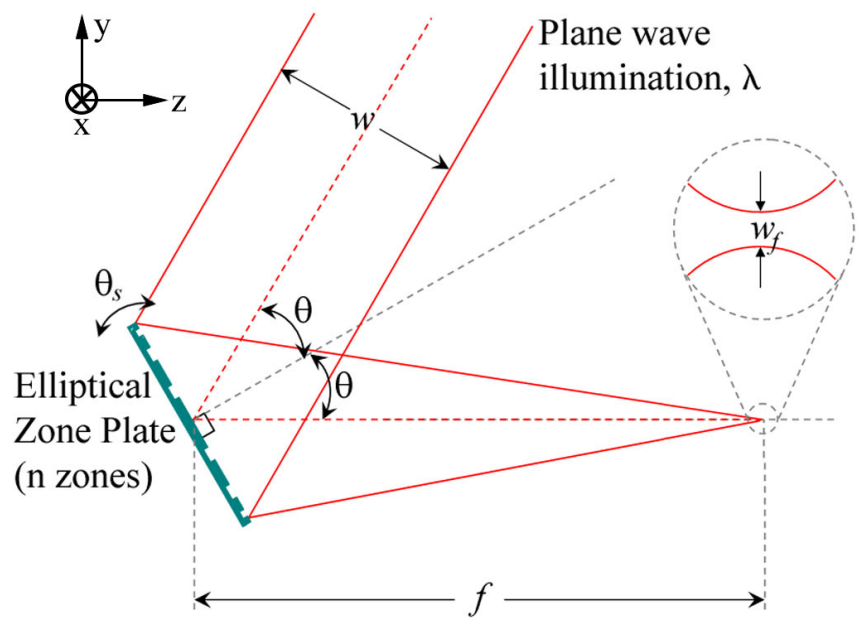

Figure 1: Illustration of design parameters for scanning binary-phase reflective Elliptical Fresnel Zone Plate (EZP). $\theta$ is the off-axis illumination angle of wavelength $\lambda, \theta_{s}$ the micromirror scan angle, $w$ and $w_{f}$ are the incident and focused beam waists, and $f$ is the focal length of the EZP with $n$ zones.

$\theta$ is the off-axis illumination angle about the $x$-axis with respect to the micromirror normal, $\lambda$ is the illumination wavelength, $f$ is the focal distance, and $a_{n}$ and $a_{n} \cos (\theta)$ are the semi-major and semiminor axes of the elliptical boundary of the $n^{\text {th }}$ zone of the EZP. Degradation in focal spot size (indicated as $w_{f}$ in Figure 1) due to changing illumination angle $\theta$ (by micromirror rotation) and wavelength are negligible for optical path difference deviation at the zone boundaries of less than $\lambda / 4$ from nominal value $[6,7]$. Therefore, the EZP can be fabricated for required focal spot size under maximum rotation angle to prevent aberrations using the following design constraints -

$$
\begin{array}{ll}
\text { Spherical Aberration: } & n \approx \sqrt{2 f / \lambda} \\
\text { Chromatic Aberration: } & n \approx \lambda / \Delta \lambda \\
\text { Off-Axis Aberration: } & \alpha \approx(3 n)^{-1 / 2} \text { (for small } n \text { ) }
\end{array}
$$

These conditions restrict the maximum number of EZP zones and micromirror scan angle (indicated as $\theta_{s}$ in Figure 1), therefore determining the number of resolvable points in the image or the "numerical aperture" of the EZP. Variation of focal distance with wavelength may provide a new mechanism for 3-D imaging through axial scan [8].

\section{FABRICATION}

The micromirror is actuated by staggered vertical comb drives fabricated by a comb self-alignment process in bonded double-SOI wafers $[9,10]$. Coarse features of the stator are etched by Deep Reactive Ion Etching (DRIE) into $25 \mu \mathrm{m}$ thick SOI $<100>$ device 


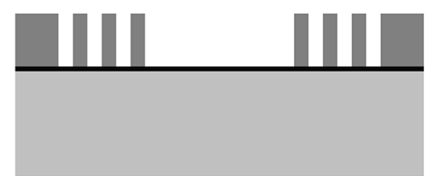

(a)

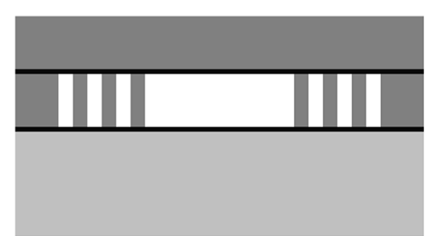

(b)

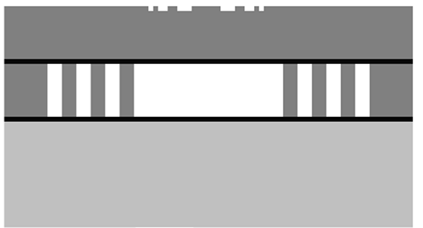

(c)

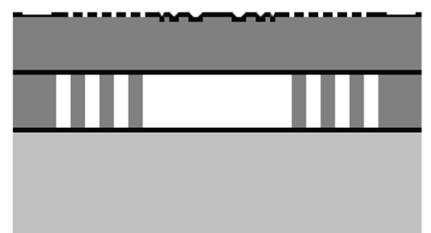

(d)

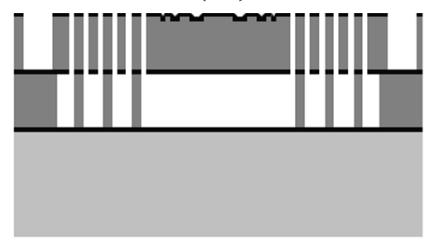

(e)

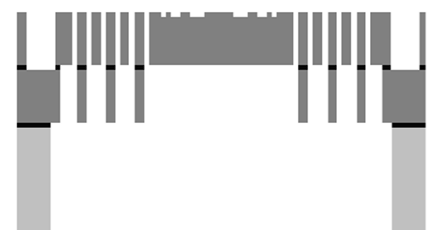

(f)
Substrate $\quad<100>$ Silicon $\square$ Silicon Dioxide

Figure 2: Device fabrication process sequence. (a) DRIE of coarse features in to SOI device layer. (b) Bond oxidized wafer, grind/polish. (c) Pattern binary-phase modulation elements of zone plate into micromirror surface. (d) Chemical vapor deposit silicon dioxide and pattern with exact micromirror features. (e) DRIE-Oxide RIE-DRIE sequence to create self-aligned actuators. ( $f$ ) Backside DRIE to release micromirror, and oxide RIE on both sides to remove protective oxide.

layer (Figure 2). An oxidized $<100>$ wafer is fusion bonded on top of the patterned wafer, and ground down to $25 \mu \mathrm{m}$ thickness with $<50 \mathrm{~nm}$ surface roughness to form the micromirror surface. Features of the elliptical zone plate objectives are patterned on the surface to quarter-wavelength depth. Exact features of the actuators, aligned to the lower layer features, are etched into deposited silicon dioxide. DRIE-oxide RIE-DRIE etching sequence forms the self-aligned. Backside substrate DRIE and oxide RIE on both sides releases the mirror and removes remaining protective oxide. Scanning electron micrographs of the fabricated device are presented in Figure 3.

\section{DEVICE CHARACTERIZATION}

Scanning EZPs designed for 635nm (1310nm) wavelength with focal lengths of $7 \mathrm{~mm}(8 \mathrm{~mm})$ and off-axis illumination angles of $20^{\circ}\left(45^{\circ}\right)$ were fabricated on micromirrors of size $500 \mu \times 700 \mu \mathrm{m}$. Two-axis beam scanning is obtained by mounting the micromirror by torsion rods within a gimbal, which is suspended by torsion rods aligned in the orthogonal direction. Rotation about each axis is driven by two sets of staggered vertical comb drives. This configuration leads to two-axis angular scanning about a single pivot point at the center of the micromirror, which reduces optical field distortions.

Frequency response characteristics (Figure 4a) of the micromirror were tested by applying voltage of $20.0+20.0 \sin (\omega t)$ volts and varying the sinusoidal frequency. The micromirror

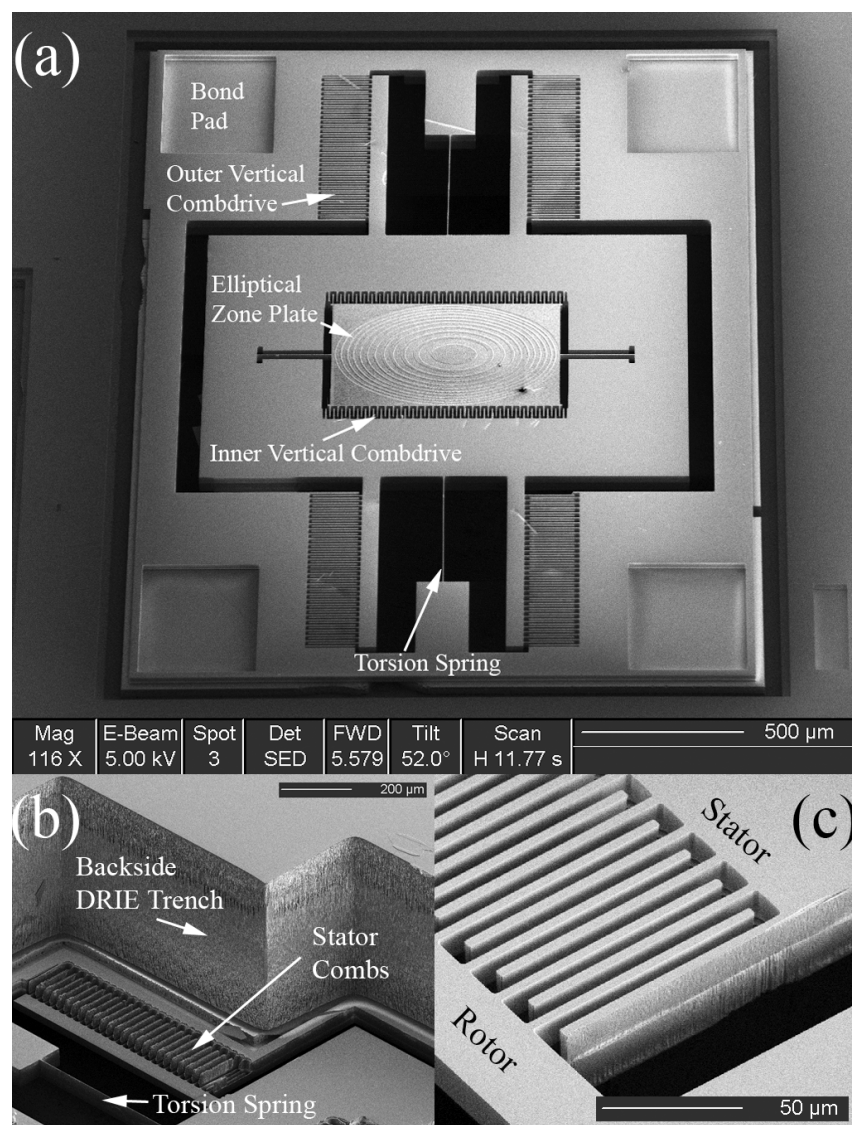

Figure 3: SEM images of the fabricated device. (a) Top view showing the EZP, vertical comb drives, torsion springs, and bond pads for electrical connection. (b) Backside view showing DRIE trench with vertical sidewalls to release the scanning micromirror. (c) Close-in view of vertical combdrive.
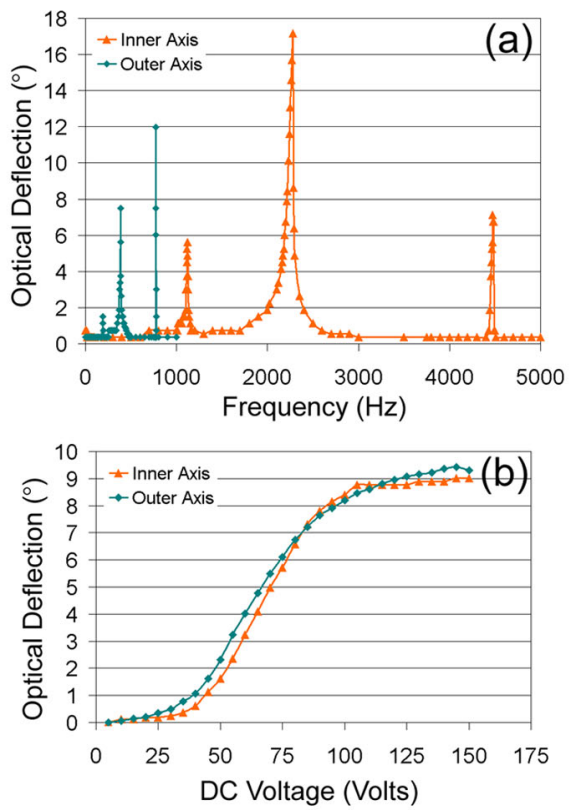

Figure 4: Micromirror operating characteristics. (a) Frequency response characteristics (Applied voltage $=20.0+$ $20.0 \sin (\omega t)$ V). (b) Static deflection characteristics on driving one comb bank on each axis. 
exhibits resonant out-of-plane rotation at $2280 \mathrm{~Hz}$ and $383 \mathrm{~Hz}$ for the inner and outer axes respectively. The static voltage deflection characteristics (Figure 4b) were determined by applying a DC voltage to one comb drive on each axis. Static voltage deflection of $\sim 9^{\circ}$ (optical) was measured on application of 110 volts on both axes. Raster scan pattern was used for point-by-point image formation, employing resonant frequency operation of the inner axis for fast line scan, and non-resonant operation of outer-axis for frame scan.

Size of the focused spot of an EZP designed with 8mm focal length for $635 \mathrm{~nm}$ illumination at $45^{\circ}$ was profiled against micromirror rotation angle by measuring the far-field angular beam divergence $(\theta)$ of the Gaussian beam, and calculating the focused beam waist $\left(w_{f}\right)$ using the formula -

$$
\theta \approx \frac{2 \lambda}{\pi w_{f}}
$$

The measured focused spot size shows little degradation (Figure 5) for micromirror scanning angles up to $10^{\circ}$ (optical) about both axes.

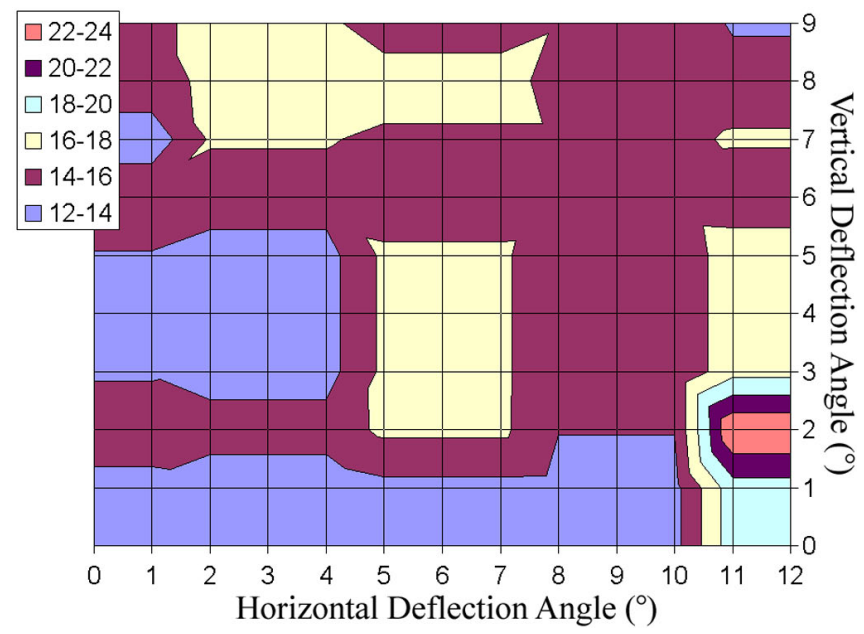

Figure 5: Map of diameter of the focused spot (in microns) created by an EZP with $8 \mathrm{~mm}$ focal length for $635 \mathrm{~nm}$ illumination at $45^{\circ}$ nominal incidence as function of optical angular deflection of the micromirror.

\section{IMAGING RESULTS}

\section{Transmission Barcode Imaging using EZP Micromirror}

Preliminary testing of image-formation capabilities of the device was performed in a simple transmission-mode experiment, depicted in figure 6. A sample with spatially-varying transmission was placed in the focal plane of a scanning EZP, and transmitted light was concentrated into a photodetector using two collection lenses. Mylar transparencies printed with longhorn logos and numbers were imaged (Figure 7) using the system at 5 frames/second. Comparison with images obtained from an Olympus BX51 microscope using 10X objective indicated an estimated field of view of $1 \mathrm{~mm} \times 0.35 \mathrm{~mm}$ at approximately $15 \mu \mathrm{m}$ resolution.

\section{EZP Micromirror-Based Reflectance Confocal Imaging}

The devices were then incorporated into a portable bench-top single-fiber laser-scanning reflectance confocal microscope

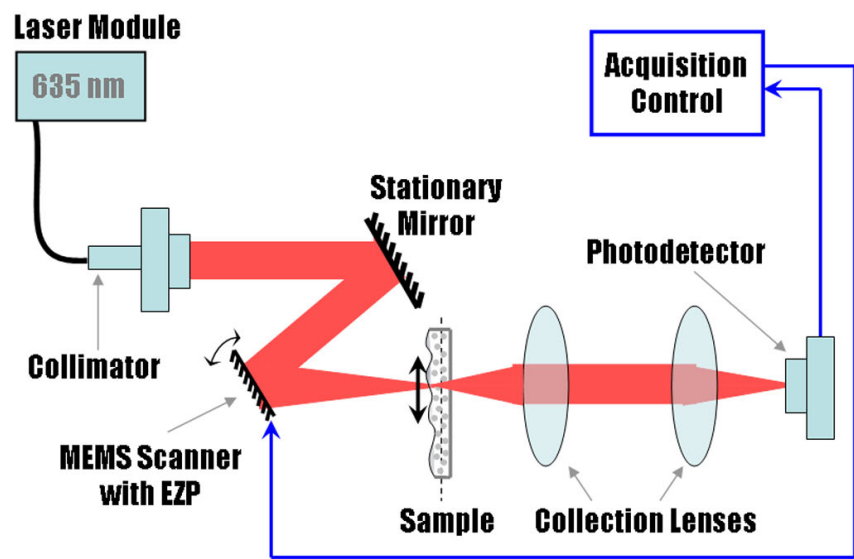

Figure 6: Schematic of transmission-mode imaging experiment used for preliminary device testing.
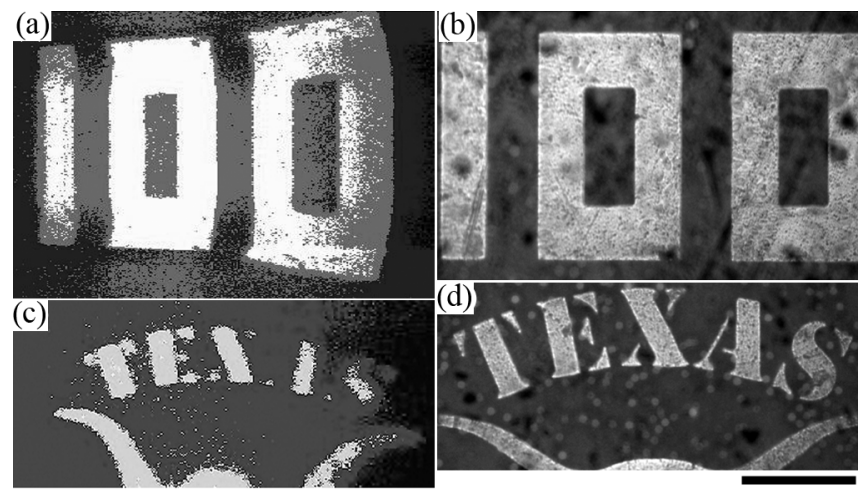

Figure 7: Results of imaging Mylar transparencies using preliminary transmission-mode imaging experiment. (a-b) Image calibration: (a) Image of number 100 (transparent) in opaque background, and (b) Image of sample using Olympus BX51 confocal microscope. (c-d) Image of longhorn symbol and text "TEXAS": (c) using the device, and (d) using Olvmpus BX51 confocal microscope. Scale Bar: 250 um.

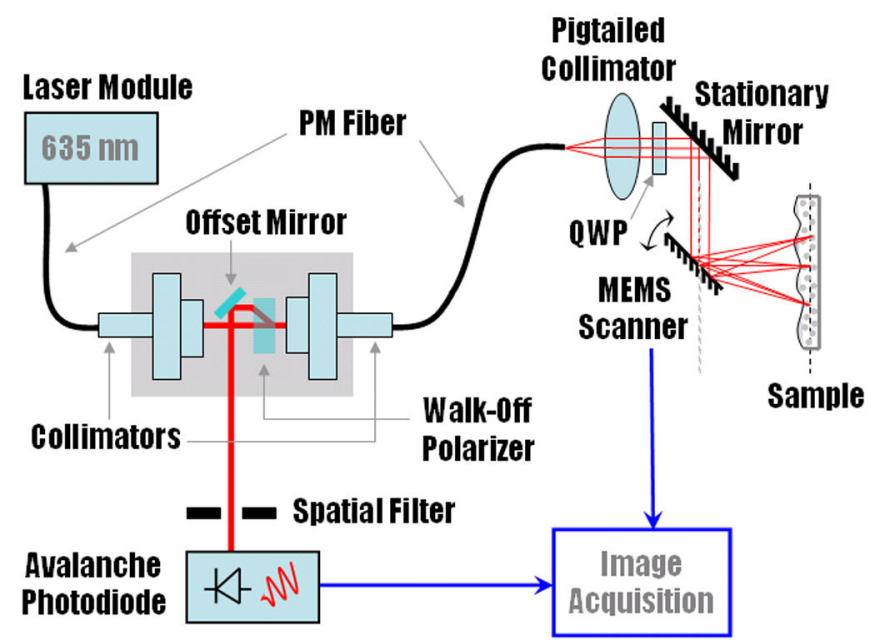

Figure 8: Schematic of single-fiber laser-scanning reflectance confocal microscope incorporating the micromirror with monolithically integrated EZP.

(Figure 8) that is better suited to eventual use in in vivo imaging applications. Polarized light from a $635 \mathrm{~nm}$ semiconductor laser 
diode is launched into a single-mode polarization-maintaining fiber, aligned to the fiber slow axis. After collimation into a beam matched to the size of the EZP, the linearly polarized light is converted into circularly polarized light by a quarter-wave plate (QWP) before being simultaneously focused and scanned across the sample by our device. Reflected light from the sample maintains some component of circularly polarized light, which is converted into light linearly polarized along the fiber fast axis, orthogonal to the incident illumination. This allows the walk-off polarizer to separate the sample reflection from laser illumination and direct it to an avalanche photodetector. Images using this system of a USAF1951 resolution target obtained are depicted in figure 9.

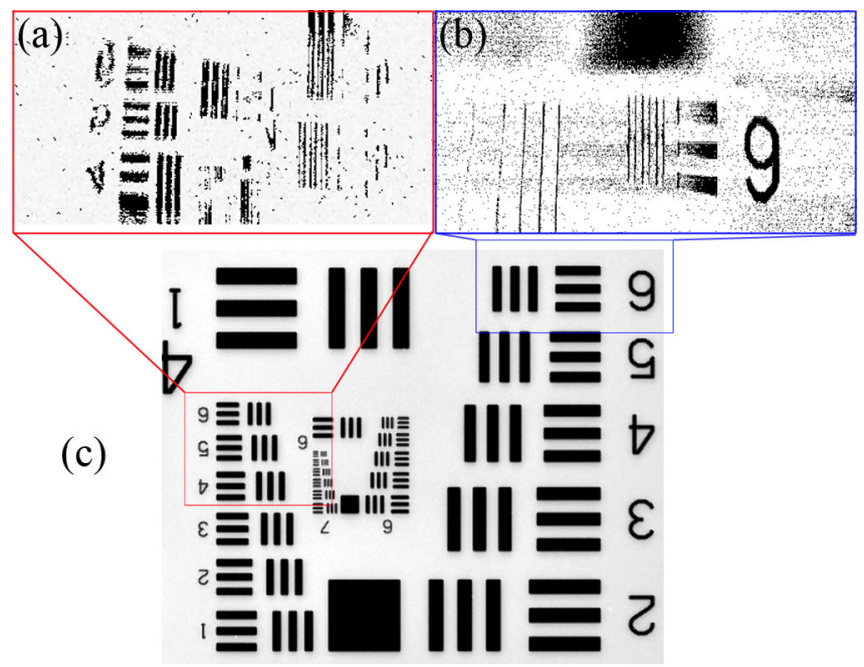

Figure 9: Results of imaging USAF1951 resolution target using laser-scanning reflectance confocal experiment using the device. Images of groups of elements from different parts of the target are depicted in (a)-(b). Field of view is $1 \mathrm{~mm} \times 0.35 \mathrm{~mm}$, and resolution is $\sim 15 \mu \mathrm{m}$. (c) Image of the target using Olympus BX51 confocal microscope with corresponding locations marked by colored rectangles.

Field of view of $1 \mathrm{~mm} \times 0.35 \mathrm{~mm}$ and lateral resolution of $15 \mu \mathrm{m}$ are estimated based on calculating the line width of the resolvable features in the resolution target images.

\section{CONCLUSIONS}

Novel monolithic integration of reflective binary-phasemodulation elements on two-axis MEMS scanning micromirrors is demonstrated for simultaneous beam scanning and focusing in a compact single-chip solution. This approach can potentially eliminate the need for focusing optics in a microendoscopes catheter, thus simplifying assembly and improving form factor. Elliptical zone plates integrated on two-axis self-aligned staggered vertical comb driven micromirrors, incorporated into a laserscanning reflectance confocal microscope experiment, were shown to provide the cellular-level resolution required in microendoscopes without complex multi-element assembly.

\section{REFERENCES}

[1] R. S. Montgomery, and S. E. Wilson, "Intraabdominal abscesses: image-guided diagnosis and therapy”, Clinical Infectious Diseases, 23, 28 (1996).

[2] Y. T. Pan, T. Q. Xie, C. W. Du, S. Bastacky, S. Meyers, and M. L. Zeidel, "Enhancing early bladder cancer detection with fluorescence-guided endoscopic optical coherence tomography”, Optics Letters, 28, 2485 (2003).

[3] Z. Yaqoob, J. Wu, E. J. McDowell, X. Heng, and C. Yang, "Methods and application areas of endoscopic optical coherence tomography”, Journal of Biomedical Optics, 11, 063001 (2006).

[4] D. Hah, P. R. Patterson, H. D. Nguyen, H. Toshiyoshi, and M. C. Wu, "Theory and experiments of angular vertical combdrive actuators for scanning micromirrors”, IEEE Journal of Selected Topics in Quantum Electronics, 10, 505 (2004).

[5] L. Y. Lin, S. S. Lee, K. S. J. Pister, and M. C. Wu, "Micromachined three-dimensional micro-optics for integrated freespace optical system”, IEEE Photonics Technology Letters, 6, 1445 (1994).

[6] M. Young, "Zone plates and their aberrations", Journal of the Optical Society of America, 62, 972 (1972).

[7] Q. Cao, and Jürgen Jahns, “Comprehensive focusing analysis of various Fresnel zone plates”, Journal of the Optical Society of America A, 21, 561 (2004).

[8] S. Cha, P. C. Lin, L. Zhu, P-C. Sun, and Y. Fainman, "Nontranslational three-dimensional profilometry by chromatic confocal microscopy with dynamically configurable micromirror scanning”, Applied Optics, 39, 2605 (2000).

[9] U. Krishnamoorthy, D. Lee, and O. Solgaard, "Self-aligned vertical electrostatic combdrives for micromirror actuation”, Journal of Microelectromechanical Systems, 12, 458 (2003).

[10] K. Kumar, K. Hoshino, H-J. Shin, R. Richards-Kortum and X. J. Zhang, "High-reflectivity two-axis vertical comb drive microscanners for confocal imaging applications”, 2006 IEEE/LEOS International Conference on Optical MEMS and Their Applications, Big Sky, MT, 8/21-24/06, pp. 120 - 121. 